\title{
MicroRNA-223 Regulates Cardiac Fibrosis After Myocardial Infarction by Targeting RASA1
}

\author{
Xiaoxiao Liu Yifeng Xu Yunfei Deng Hongli Li \\ Department of Cardiology, Shanghai General Hospital, School of Medicine, Shanghai Jiao Tong \\ University, Shanghai, China
}

\section{Key Words}

Microrna-223 • Cardiac fibrosis • RASA1 • Myocardial infarction

\begin{abstract}
Background/Aims: Percutaneous coronary intervention reduces acute myocardial infarction (MI)-induced mortality to a great extent, but effective treatments for MI-induced cardiac fibrosis and heart failure are still lacking. MicroRNAs (miRNAs) play a variety of roles in cells and have thus been investigated extensively. MicroRNA-223 (miR-223) expression has been reported to be altered in post-MI heart failure in humans; however, the roles of miR-223 in MI remain unknown. Our study aimed to elucidate the roles of miR-223 in cardiac fibrosis. Methods: Cultured cardiac fibroblasts (CFs) were activated by TGF- $\beta 1$ stimulation. Gain and loss of miR-223 and RAS p21 protein activator 1 (RASA1) knockdown in CFs were achieved by transfecting the cells with miR-223 mimics and inhibitors, as well as small interfering RNA-RASA1 (siRASA1), respectively. Quantitative real-time reverse transcriptase-polymerase chain reactions (qRT-PCR) was used to determine miR-223-3p and RASA1 expression levels, and Cell Counting Kit-8 (CCK-8), transwell migration and scratch assays were performed to assess CFs viability and migration, respectively. Western blotting was used to detect collagen I, collagen III, alpha-smooth muscle actin (a-SMA), RASA1, p-Akt/t-Akt, p-MEK1/2/t-MEK1/2, and $\mathrm{p}$-ERK1/2/t-ERK1/2 protein expressions, and immunofluorescence assays were used to detect the expression of $\alpha$-actin, vimentin and $\alpha$-SMA. Luciferase assays were carried out to determine whether miR-223 binds to RASA1. Rat models of MI were established by the ligation of the left anterior descending (LAD) coronary artery. MiR-223 inhibition in vivo was achieved via intramyocardial injections of the miR-223 sponge carried by adeno-associated virus 9 (AAV9). The cardiac function was detected by echocardiography, and cardiac fibrosis was shown by Masson's trichrome staining. Results: miR-223 was increased in CFs compared to cardiomypcytes, and TGF- $\beta 1$ treatment increased miR-223 expression in CFs. The miR-223 mimics enhanced cell proliferation and migration and collagen I, collagen III, and $\alpha$-SMA protein expression in CFs, while the miR-223 inhibitors had contrasting effects and partially prevented the promoting effects of TGF- $\beta 1$. qRT-PCR and western blotting revealed that miR223 negatively regulated RASA1 expression, and the luciferase assays showed that miR-223
\end{abstract}

Xiaoxiao Liu and Yifeng Xu contributed equally to this work. 


\section{Cellular Physiology Cell Physiol Biochem 2018;46:1439-1454

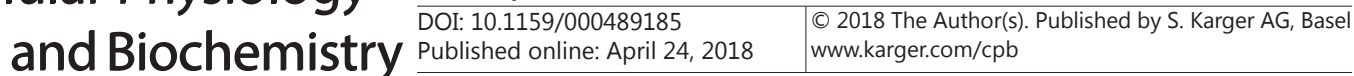

Liu et al.: The Roles of MicroRNA-223 in Cardiac Fibrosis

suppressed the luciferase activity of the RASA1 3' untranslated region (3'UTR), indicating that miR-223 binds directly to RASA1. Similar to transfection with the miR-223 mimics, RASA1 knockdown enhanced cell proliferation and migration and collagen I, collagen III, and $\alpha$-SMA protein expression in CFs. Moreover, RASA1 knockdown partially reversed the inhibitory effects of the miR-223 inhibitor on cell proliferation and migration and collagen I, collagen III, and $\alpha$-SMA protein expression, indicating that the effects of miR-223 in CFs are partially mediated by the regulation of RASA1 expression. Further exploration showed that miR-223 mimics and siRASA1 promoted MEK1/2, ERK1/2 and AKT phosphorylation, while the miR-223 inhibitors had contrasting effects. The in vivo experiments confirmed the results of the in vitro experiments and showed that miR-223 inhibition prevented cardiac functional deterioration and cardiac fibrosis. Conclusions: miR-223 enhanced cell proliferation, migration, and differentiation in CFs, thus mediated cardiac fibrosis after MI partially via the involvement of RASA1.

(C) 2018 The Author(s)

Published by S. Karger AG, Basel

\section{Introduction}

Acute myocardial infarction (AMI) remains a major cause of morbidity and mortality in humans. The arrest of the flow of blood and oxygen to the heart causes cardiomyocyte loss, as well as pathological remodeling and reductions in myocardial function, changes that inevitably lead to heart failure. When AMI occurs, cardiac fibroblasts (CFs) proliferate, differentiate into myofibroblasts and produce excessive extracellular matrix to fill in the gaps created by the removal of dead cells, as cardiomyocytes are nonrenewable. Diffuse interstitial fibrosis, a key morphological change that occurs as part of the structural myocardial remodeling that takes place after AMI, disturbs the collagen fiber network of the myocardium [1-3], resulting in abnormal myocardial stiffness, which contributes to the development of ventricular dysfunction, arrhythmias and heart failure. Therefore, inhibiting cardiac fibrosis may be clinically important with respect to the prevention and treatment of heart failure after MI. Specific anti-fibrotic agents are not currently available; thus, the development of novel molecular therapies for the treatment of cardiac fibrosis remains an important issue.

MicroRNAs (miRNAs) are a class of endogenous small non-coding RNAs that repress gene expression by degrading or inhibiting the translation of target mRNAs by acting on their 3 ' untranslated regions (3'UTRs) [4]. miRNAs have been implicated in various biological and pathological phenomena, including cardiovascular diseases [5, 6]. MicroRNA-223 (miR-223) has recently attracted large amounts of attention in cardiovascular biology and has been implicated in different cardiac pathological processes, including cardiomyocyte hypertrophy, necrosis and glucose metabolism abnormalities, in several studies. In a previous study, Van Rooij et al. found that miR-223 expression was markedly increased in the border zones of infarcted myocardial tissues in humans with post-MI heart failure [7]. Moreover, Morishita et al. showed that miR-223 expression was increased by more than 2 fold compared with its baseline level in rat peritoneal fibrosis [8], indicating that miR-223 participates in fibrosis. However, the effects of miR-223 on CFs proliferation and function and myocardial fibrosis, as well as the mechanisms by which it exerts its effects, are largely unknown.

Evidence indicates that the RAS signaling pathways play important roles in various cardiac diseases $[9,10]$. RAS serves as a molecular microswitch in these pathways, and RAS proteins transition between RASGDP (inactivated states) and RASGTP (activated state) states. When the RAS protein combines with RAS GTPase-activating proteins (RASGAPs), the intrinsic GTPase activity of RAS increases by more than 100000 fold, leading to the hydrolysis of RASGTP to RASGDP, which results in the inactivation of the RAS protein and RAS signaling [11]. RASA1 (RAS p21 protein activator (GTPase-activating protein)), which is also known as p120RasGAP, was the first RasGAP protein identified by researchers and acts mainly by negatively regulating RAS signaling. Recent studies have shown that the RAS signaling pathways may have common pro-fibrotic effects [12-14]. Ana Maria Queirós et al. 
Liu et al.: The Roles of MicroRNA-223 in Cardiac Fibrosis

proposed that miRNAs targeting RAS signaling pathway repressor, like RASA1, RASA2, may promote cardiac fibrosis [15]. Moreover, Li et al. revealed that silencing RASA1 expression promoted human dermal fibroblast migration [16]. Thus, we hypothesized that RASA1 also participates in the pathogenesis of cardiac fibrosis. Previous studies showed that RASA1 is a target gene of miR-223 [17, 18]; however, whether miR-223 regulates RASA1 in CFs remains unknown.

In this study, we found that miR-223 overexpression promoted cell proliferation, migration, and differentiation in cultured CFs, whereas miR-223 inhibition had contrasting effects. In addition, the in vivo experiments showed that the miR-223 sponge mediated by adeno-associated virus 9 (miR-223 sponge AAV9) prevented post-MI heart failure-induced miR-223 upregulation and thus improved cardiac function and significantly alleviated cardiac fibrosis. Further study indicated that RASA1 was a target gene of miR-223 in CFs and that RASA1 and its downstream pathways mediated the pro-fibrotic effects of miR-223.

\section{Materials and Methods}

\section{Animal Models}

Male Sprague-Dawley rats (SD) (180 to 200 g) were randomly divided into the following 4 groups: a sham group, an MI group, an (MI+miR-223 sponge AAV9) group, and an (MI+miR-scramble) group. The MI model was induced as described previously [19]. Briefly, the rats were anesthetized with intraperitoneal sodium pentobarbital (50 mg/kg), intubated, and then artificially ventilated with a rodent ventilator. The rats underwent thoracotomy and pericardiotomy, and then the left anterior descending (LAD) coronary artery was ligated at its origin by a 6-0 prolene suture. The rats in the sham group underwent the same procedure as the rats in the experimental groups; however, they were not subjected to LAD coronary artery ligation. Adeno-associated virus (AAV) is an efficient and safe vehicle for the performance of in vivo gene transfer experiments, and serotype 9 is significantly cardiotropic and has been widely used [20-22]. To investigate whether miR-223 inhibition is sufficient to prevent cardiac fibrosis in vivo, we intramyocardially injected the rats in the (MI+miR-223 sponge AAV9) group with the miR-223 sponge AAV9 (Obio Technology, Shanghai, China) at a dose of $5^{*} 10^{11}$ viral genomes (vg) per animal before LAD ligation. We injected the rats in the (MI+miR-scramble) group with miR-scramble. After 8 weeks, all the rats underwent echocardiographic evaluations, during which their cardiac function was assessed. The rats were then sacrificed by cervical dislocation so that their tissues could be used in subsequent experiments. All animal experimental protocols were approved by the Animal Care and Use Committee, Research Institute of Medicine, Shanghai Jiao Tong University, and all animal experiments were conducted in accordance with the guidelines of the National Institutes of Health, 8th edition, 2011. All efforts were made to minimize animal suffering.

\section{Primary cells isolation and culture}

The primary cells used herein were isolated as previously described [23]. Briefly, SD rats aged 1 to 3 days were euthanized by decapitation, after which the hearts were removed, and the ventricles were finely minced and digested in an enzyme mixture (the ratio of $0.25 \%$ trypsin to $0.1 \%$ collagenase was $1: 1$ ). The cell suspensions were collected and centrifuged, and then the cells were resuspended in Dulbecco's Modified Eagle Media (DMEM, Gibco, Grand Island, CA, USA) with 10\% fetal bovine serum (Gibco, Grand Island, CA, USA), $100 \mathrm{U} / \mathrm{ml}$ penicillin and $100 \mu \mathrm{g} / \mathrm{ml}$ streptomycin. The cells were then incubated for 1.5 hours under standard culture conditions $\left(37^{\circ} \mathrm{C}\right.$ in $\left.5 \% \mathrm{CO} 2\right)$, which allowed the fibroblasts to attach to the culture plates. On the other hand, cardiomyocytes rarely attached until at least 24 hours. Thus we harvested cardiomyocytes and CFs according to their different attachment times. Almost all the CFs used in our experiments were thin and triangular, while cardiomyocytes were beating cells. CFs from passage 2 (P2) and cardiomyocytes cultured for three days were used for subsequent experiments.

\section{MiRNA transfection and RNA interference}

To achieve the gain or loss of miR-223 expression and silence RASA1 expression, we transfected miR-223 mimics (50 nM), miR-223 inhibitors (100 nM), and small interfing RNA-RASA1 (siRASA1, $50 \mathrm{nM}$ ) (RiboBio, Guangzhou, China), respectively, into CFs using Lipofectamine 3000 (Invitrogen, USA, California), according 


\section{Cellular Physiology Cell Physiol Biochem 2018;46:1439-1454 and Biochemistry Published online: April 24, $2018 \quad \begin{aligned} & \text { DOI: 10.1159/000489185 } 2018 \text { The Author(s). Published by S. Karger AG, Basel } \\ & \text { www.karger.com/cpb }\end{aligned}$}

Liu et al.: The Roles of MicroRNA-223 in Cardiac Fibrosis

to the manufacturer's instructions. Control cells were transfected with the appropriate negative controls. The sequences of the oligonucleotides were as follows: miR-223 mimic: 5'-UGUCAGUUUGUCAAAUACCCC-3'; miR-223 inhibitor: 5'-ACAGUCAAACAGUUUAUGGGG-3'; and siRASA1: 5'- TAGGATATTACAGTCACGT-3'.

\section{Quantitative real-time RT-PCR}

To measure mRNA and miRNA expression, we extracted total RNA from cardiac tissues and cultured cells using Trizol reagent (Invitrogen USA). RNA quality and purity were measured using a NanoDrop-2000 spectrophotometer. To determine miRNA expression levels, we synthesized cDNA with a special stem-loop primer specific for the target miRNA. Target miRNA expression was then quantified by quantitative real-time reverse transcriptase-polymerase chain reactions (qRT-PCR), which was performed with the appropriate forward and reverse primers and a microRNA qRT-PCR SYBR Green Detection Kit (BioTNT, Shanghai, China), according to the manufacturer's instructions. For the RASA1 mRNA quantification experiments, we used a SYBR RT-PCR kit (Takara Bio Inc., Japan) to synthesize cDNA and perform qRT-PCR analysis. RNA expression was quantified using the appropriate comparative cycle threshold (CT) values. U6 and GAPDH served as internal controls. The expression levels of miRNA and mRNA are presented as fold changes relative to the expression levels of a control and were calculated by the 2- $\Delta \Delta \mathrm{Ct}$ method. The following primers were used for the experiment: rat miR-223 primer for RT: CTCAACTGGTGTCGTGGAGTCGGCAATTCAGTTGAGGGGGTAT;

forward: 5'-TGT CAG TTT GTC AAA TAC C-3'; reverse: 5'-AACTGGTGTCGTGAG-3'; rat U6 primer for RT: CTCAACTGGTGTCGTGGAGTCGGCAATTCAGTTGAGAAAATATG; forward: 5'-CAA ATT CGT GAA GCG TT-3'; reverse: 5'-TGG TGT CGT GGA GTCG-3'; rat GAPDH forward: 5-ACGGCAAGTTCAACGGCAC-3'; rat GAPDH reverse: 5'-CGCCAGTAGACTCCACGACATA-3'; and rat RASA1 forward: 5'-CTGGAGATTATTCCCTGTATTTTCG-3'; rat RASA1 reverse: 5'-TGTTCTTTCCGATAGTGGTCTATGA-3'.

\section{Western blot analysis}

Total protein was extracted from myocardial tissues or CFs. The proteins were separated by SDS/PAGE before being transferred to PVDF membranes, which were blocked and then incubated with the appropriate primary antibodies overnight at $4^{\circ} \mathrm{C}$. The membranes were incubated with a horseradish peroxidaseconjugated secondary antibody, and then the antibody complexes were visualized and quantified using ECL chemiluminescence Kit (Yeasen, China) and a chemiluminescence-western blotting detection system (Tanon, Shanghai, China). The following primary antibodies were used in this experiment: anti-RASA1 (1:1000, Abcam, Cambridge, Britain), anti-collagen I (1:1000, Abcam, Cambridge, Britain), anti-collagen III (1:1000, Abcam, Cambridge, Britain), anti- $\alpha$-SMA (1:1000, Cell Signaling Technology, USA), anti-p-ERK (1:1000, Cell Signaling Technology, USA), anti-t-ERK (1:1000, Cell Signaling Technology, USA), anti-p-AKT (1:1000, Cell Signaling Technology, USA), anti-t-AKT (Cell Signaling Technology, USA), p-MEK (Cell Signaling Technology, USA), anti-t-MEK (Cell Signaling Technology, USA), and anti-GAPDH (1:1000, ProteinTech, USA). HRP-labeled goat anti-rabbit IgG and HRP-labeled goat anti-mouse IgG were purchased from Beyotime (China). The protein expression levels of the target genes were quantified by relative densitometry and normalized to the protein expression levels of GAPDH, which was used as an internal control. The expression levels of the proteins are presented as fold changes relative to those of a control sample.

\section{CF proliferation assay}

Cell proliferation was assessed with Cell Counting Kit-8 (CCK-8) assay, according to the manufacturer's instructions. CFs were transfected for 6 hours, after which they were digested with $0.25 \%$ trypsin and plated in 96-well plates at a density of 4000 cells/plate. After the treatments and the designated amount of time had passed, the culture medium was replaced with $110 \mu$ l of CCK- 8 solution (containing $100 \mu$ l of serum-free DMEM and $10 \mu \mathrm{l}$ of CCK- 8 reagent). After the cells had incubated for 4 hours, we measured the absorbance at a wavelength of $450 \mathrm{~nm}$ with a microplate spectrophotometer. There are 6 replicates in each individual experiment among three repeated experiments.

\section{Transwell migration assay}

Transwell migration assay was performed using a BD Chamber (BD Falcon). After treatment, $5 \times 10^{4}$ cells suspended in serum-free DMEM were placed in the upper chamber of the insert. Medium containing $10 \%$ FBS was added to the lower chamber of the insert. Twenty-four hours thereafter, the cells that had migrated through the membrane were stained with $0.1 \%$ crystal violet and counted under a microscope. 


\section{Cellular Physiology Cell Physiol Biochem 2018;46:1439-1454 \\ \begin{tabular}{ll|l} 
and Biochemistry & $\begin{array}{l}\text { Dublished online: April 24, } 2018 \\
\text { Puld } 2018 \text { The Author(s). Published by S. Karger AG, Basel } \\
\text { www.karger.com/cpb }\end{array}$ \\
\hline
\end{tabular}}

Liu et al.: The Roles of MicroRNA-223 in Cardiac Fibrosis

Migrated cell numbers were counted in 5 random fields per chamber in each experiment. The CFs capacity of migration was expressed by the migrated cells counted normalized to the corresponding controls.

\section{Scratch assay}

For the scratch assay, CFs from P2 were plated in a 6-well plate. After designed treatments, a linear wound was introduced into the CFs monolayer with a 1-ml micropipette tip. The cells were then washed with PBS and cultured in fresh complete medium for 24 hours. The sizes of the wounds were determined by microscopy after 24 hours. The widths at 0,24 hours were measured, and migration rates [(scratch width at 0 hour - scratch width at 24 hours)/scratch width at 0 hour $\times 100 \%$ ] were calculated. Mean values were obtained from at least 3 separate experiments and normalized to the corresponding controls.

\section{Immunofluorescence assay}

CFs were plated in 35-mm glass dishes and underwent the designed treatments. The cells were washed with PBS and then fixed with 4\% paraformaldehyde for 20 minutes at room temperature. After being washed thrice with PBS, the CFs were blocked in 5\% goat serum for 1 hour. The cells were subsequently incubated with primary antibodies specific for $\alpha$-actin (1:100, CST), or vimentin (1:200, CST), or $\alpha$-SMA (1:500, Abcam) overnight at $4^{\circ} \mathrm{C}$. The next day, the CFs were washed with PBS, after which they were incubated with fluorescence- conjugated secondary antibodies (Beyotime, China), followed by DAPI. Images were captured under a fluorescence microscope.

\section{Dual-luciferase reporter assay}

The RASA1 gene was predicted to be a target gene of miR-223 by bioinformatics software. To construct the luciferase reporter vector and form a RASA1 3'UTR wild-type (WT) luciferase vector, we synthesized RASA1 3'UTR fragments containing the predicted miR-223 binding site (Fig. 4E) and inserted the fragments into a pmirGLO dual-luciferase miRNA target expression vector. The RASA1 3'UTR mutant-type (MUT)luciferase vector, whose miR-223 binding region contained a point-mutated sequence, was generated using a MutaBest kit. For the reporter assay, CFs seeded in 24-well plates were cotransfected with the luciferase reporter construct and miR-223 mimics or negative control miRNA. After undergoing transfection for forty-eight hours, the cells were lysed, and luciferase activity levels were measured using a dual-luciferase reporter assay system. Firefly luciferase activity levels were normalized to Renilla luciferase activity levels and the luciferase activity are presented as fold changes relative to the control group.

\section{Masson's trichrome staining}

The border zones of infarcted myocardial tissues from MI rats and left ventricular myocardial tissues from sham group rats were separated, fixed in $4 \%$ formaldehyde and then embedded in paraffin. The tissues were sectioned, after which they were stained with Masson trichrome (Sigma-Aldrich), according to the manufacturer's instructions, to detect collagen deposition. The collagen fibers stained blue, the cell nuclei stained black, and the cell cytoplasm stained red.

\section{Statistical analysis}

The experiments were repeated at least three times, and the values are expressed as the mean \pm SD. Differences between two groups were evaluated with Student's t-test, and multiple comparisons were performed using one-way ANOVA. $\mathrm{P}<0.05$ was considered significant. All statistical analyses were performed using GraphPad Prism 5.0 (GraphPad Software).

\section{Results}

\section{miR-223 is increased in activated CFs}

To figure out the miR-223 expression patterns in cardiac major cell types, we conducted qRT-PCR assay to detect the relative expression levels of miR-223 in isolated CFs and cardiomyocytes from neonatal rat ventricles. Fig. 1A exhibited the identifications of cardiomyocytes and CFs. As presented, the expression of $\alpha$-actin and vimentin were used as markers to determine the purity of cardiomyocytes and fibroblasts, respectively. The purity 
Fig. 1. miR-223 is increased in activated CFs. (A) Identifications of neonatal rat cardiomyocytes (NRCMs) and neonatal rat cardiac fibroblasts (NRCFs). a-actin, the marker of NRCMs, was detected by immunofluorescence using anti-a-actin antibody, Alexa Fluor 594--conjugated goat anti-mouse secondary antibody (red staining), and DAPI for the nuclei (blue). Vimentin, the marker of NRCFs, was detected by immunofluorescence using antivimentin antibody, FITC-conjugated goat anti-mouse secondary antibody (green staining), and DAPI for nuclei (blue). Undur 100x microscope. Scale bar: $100 \mu \mathrm{m}$. (B) Expression of miR-223 in NRCFs compared to NRCMs, $\mathrm{n}=3$. ${ }^{*} \mathrm{P}<0.05$ versus NRCMs. (C) CFs were stimulated with TGF- $\beta 1$ with different concentrations $(0,5,10 \mathrm{ng} / \mathrm{ml})$ for 48 hours. Relative $\alpha$-SMA protein levels were quantified and shown as the mean $\pm \mathrm{SD}, \mathrm{n}=3 .{ }^{* *} \mathrm{P}<0.01,{ }^{* * *} \mathrm{P}<0.001$ versus control. \#\#\#P<0.001 versus TGF- $\beta 1$ ( $5 \mathrm{ng} / \mathrm{ml})$. (D) Expression of miR-223 in CFs stimulated with TGF- $\beta 1$ with different concentrations for 24 hours, $\mathrm{n}=3 .{ }^{*} \mathrm{P}<0.01$, ${ }^{* * *} \mathrm{P}<0.001$ versus control. $\# \mathrm{P}<0.001$ versus $\mathrm{TGF}-\beta 1$ $(5 \mathrm{ng} / \mathrm{ml})$.

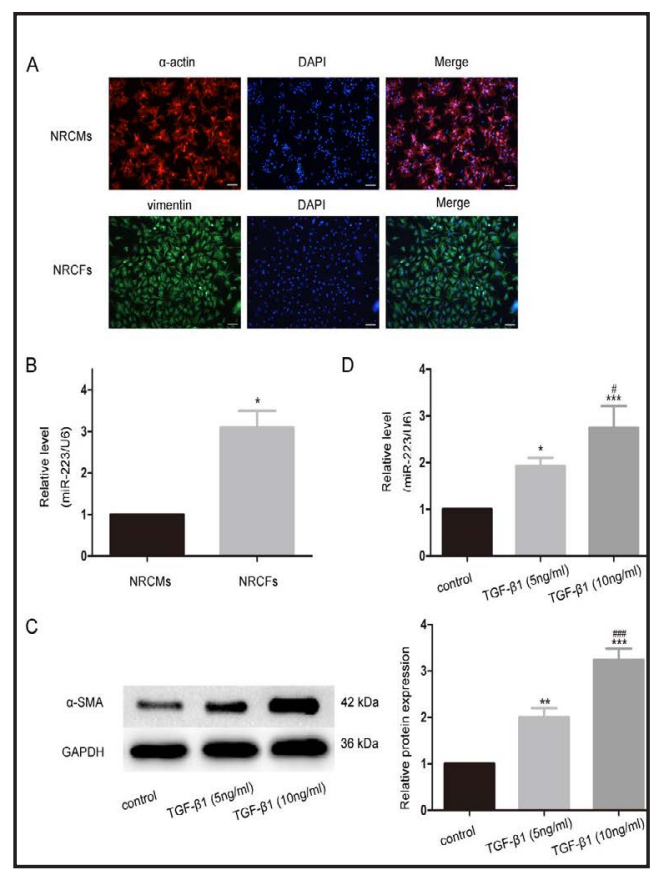

of cardiomyocytes or fibroblasts used in our experiments is more than $90 \%$. We observed that the miR-223 expression levels in CFs were remarkably higher than those in cardiomyocytes (Fig. 1B). Moreover, we stimulated the differentiation of CFs into myofibroblasts by TGF- $\beta 1$ with increasing concentration. Protein expression of $\alpha$-SMA, the myofibroblasts marker, was increased in the concentration-dependent manner (Fig. 1C). Most importantly, miR-223 was also increased in the stimulated CFs (Fig. 1D). Taken together, these data supported a potential role for miR-223 in cardiac fibrosis.

The miR-223 mimic promotes CFs proliferation, migration, and myofibroblast differentiation in vitro, while the miR-223 inhibitor has contrasting effects

Activated CFs, whose proliferation and migration abilities, collagen synthesis abilities, $\alpha$-SMA expression levels and myofibroblast differentiation abilities are enhanced, play vital roles in post-MI cardiac remodeling and heart failure. Thus, we detected the effects of miR-223 on CFs activation by transfecting CFs with miR-223 mimics or inhibitors to overexpress or knock down miR-223, respectively. qRT-PCR analysis, whose results are shown in Fig. 2A, revealed that miR-223 expression levels were increased in the miR-223mimic group and decreased in the miR-223-inhibitor group remarkably compared with the corresponding control groups, indicating that the efficiency which the mimics and inhibitors were transfected was high. We then conducted CCK-8, transwell migration and scratch assays. The CCK assay showed that the miR-223 mimic promoted CFs proliferation in a timedependent manner, and the transwell and scratch assays showed that the miR-223 mimic promoted CFs migration after 48 hours of transfection. However, the miR-223 inhibitor had contrasting effects (Fig. 2B, C, D). Western blot analysis indicated that the miR-223 mimic augmented collagen I, collagen II and $\alpha$-SMA expression in CFs, while the miR-223 inhibitor suppressed the expression of these proteins (Fig. 2E). We also analyzed the expression of $\alpha$-SMA, the myofibroblast marker, by immunofluorescence assay. The results (Fig. 2F), which were similar to those of the western blotting experiments, showed that the miR-223 mimic elicited enhancements of the fluorescence intensity, while the miR-223 inhibitor weakened the fluorescence intensity compared with the corresponding control treatments. Therefore, the miR-223 mimic promoted CFs proliferation, migration, and myofibroblast differentiation in vitro, while the miR-223 inhibitor had contrasting effects. 
Fig. 2. The miR-223 mimic promotes CFs proliferation, migration, and myofibroblast differentiation in vitro, while the miR-223 inhibitor has contrasting effects. CFs were transfected with miR-223 mimic, miR-223 inhibitor or the corresponding negative controls (mimic nc or inhibitor nc) and then subjected to the following assays. (A) qRT-PCR analysis of miR-223 expression in cultured CFs transfected for 24 hours, $n=3$. (B) CCK8 assay showing CFs proliferation at different times $(24,48$, 72, 96 hours) after transfection, $n=3$. (C) After transfection for 48 hours, CFs were digested with trypsin and then $50000 \mathrm{CFs}$ in each group were plated in the upper chambers of tranwell culture plate. After 24 hours, the migrated CFs were dyed and culpture under $100 \mathrm{x}$ microscope. Scale bar: $100 \mu \mathrm{m}, \mathrm{n}=3$. (D) Scratch assays showing the migration ability of CFs after transfection for 48 hours, under 100x microscope. Scale

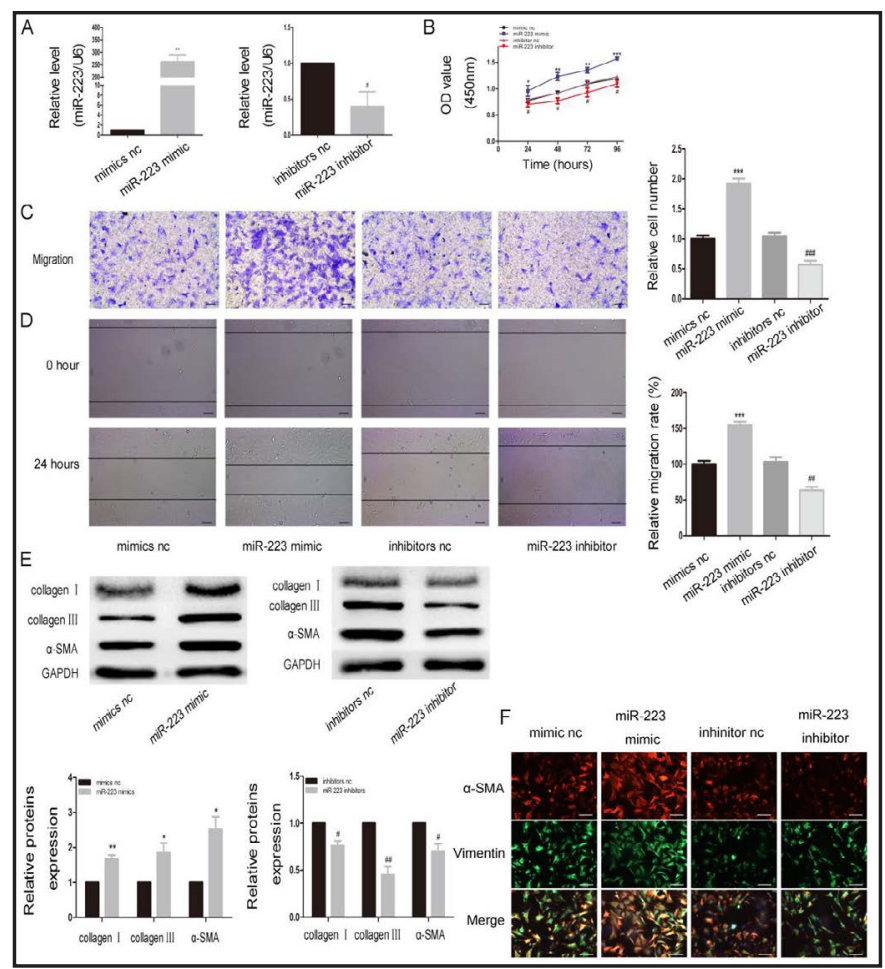
bar: $100 \mu \mathrm{m}, \mathrm{n}=3$. (E) Representative western blotting results and quantitative data pertaining to collagen I, collagen III, and $\alpha$-SMA expression in CFs after transfection for 48 hours, $n=3$. (F) Immunofluorescence images showing the effects of each type of transfection for 48 hours on the fluorescence intensity. a-SMA was detected by immunofluorescence using anti-a-SMA antibody, Cy3-conjugated goat anti-rabbit secondary antibody (red staining). Vimentin was detected by immunofluorescence using anti-vimentin antibody, FITCconjugated goat anti-mouse secondary antibody (green staining), and DAPI for nuclei (blue). Under 200x microscope. Scale bar: $100 \mu \mathrm{m}, \mathrm{n}=3 .{ }^{*} \mathrm{P}<0.05,{ }^{* *} \mathrm{P}<0.01,{ }^{* * *} \mathrm{P}<0.001$ versus mimic nc. $\# \mathrm{P}<0.05, \# \# \mathrm{P}<0.01$, \#\#\#P<0.001 versus inhibitor nc.

\section{miR-223 mediates the pro-fibrotic effects of TGF- $\beta 1$ in vitro}

As we have presented above, miR-223 is increased in TGF- $\beta 1$-stimulated CFs and overexpression of miR-223 promotes CFs proliferation, migration, and myofibroblast differentiation itself. Then we aimed to investigate whether miR-223 is a downstream effector of TGF- $\beta 1$ in CFs. We transfected CFs with miR-223 inhibitors or corresponding controls before TGF- $\beta 1$ treatment. qRT-PCR assay showed that miR-223 inhibitors downregulated the expression of miR-223 successfully compared with the controls under TGF- $\beta 1$ treatment (Fig. 3A). CCK8 and transwell assays presented that TGF- $\beta 1$ treatment remarkably facilitated the proliferation and migration of CFs, while pre-transfecting CFs with miR-223 inhibitors evidently alleviated the effects of TGF- $\beta 1$ (Fig. 3B, C). Moreover, miR-223 inhibitors prevented the enhancement of collagen I, collagen III, and $\alpha$-SMA protein expressions under TGF- $\beta 1$ treatment (Fig. 3D), which indicated that miR-223 knockdown inhibited myofibroblast differentiation stimulated by TGF- $\beta 1$ as well. The immunofluorescence results varified the $\alpha$-SMA protein expressions under different treatments (Fig. 3E). As a result, miR-223 may play a mediation role in the pro-fibrotic process of TGF- $\beta 1$.

\section{RASA1 is a direct target of miR-223 in CFS}

To elucidate the mechanisms underlying the above phenomena, we attempted to identify the target genes of miR-223. The results of a bioinformatics analysis performed by TargetScan predicted that the 3'UTR of RASA1 possesses a target site for miR-223. Therefore, we investigated whether miR-223 targets RASA1. The results showed that the miR-223 mimic 
Fig. 3. miR-223 mediates the pro-fibrotic effects of TGF- $\beta 1$ in vitro. We transfected CFs with miR223 inhibitors or corresponding controls for 24 hours and then treated the transfected CFs with $10 \mathrm{ng} / \mathrm{ml}$ TGF- $\beta 1$ for appointed time to Fig. out whether knockdown of miR-223 could reverse the effects of TGF- $\beta 1$ on CFs. (A) qRT-PCR analysis of miR-223 expression after treating the transfected CFs with TGF- $\beta 1(10 \mathrm{ng} / \mathrm{ml})$ for 24 hours, $\mathrm{n}=3$. (B) CCK8 assay showing the cell proliferation of CFs after treating the transfected CFs with TGF- $\beta 1(10 \mathrm{ng} / \mathrm{ml})$ for 48 hours, $\mathrm{n}=3$. (C) After treating the transfected CFs with TGF- $\beta 1$ (10ng/ $\mathrm{ml}$ ) for 48 hours, CFs were digested with trypsin and then $50000 \mathrm{CFs}$ in each group were plated in the upper chambers of tranwell culture plate. After 24 hours, the migrated CFs were dyed and culpture under 200x microscope. Scale bar: $50 \mu \mathrm{m}$, $\mathrm{n}=3$. (D) Representative western blotting results and quantitative data pertaining to collagen I,

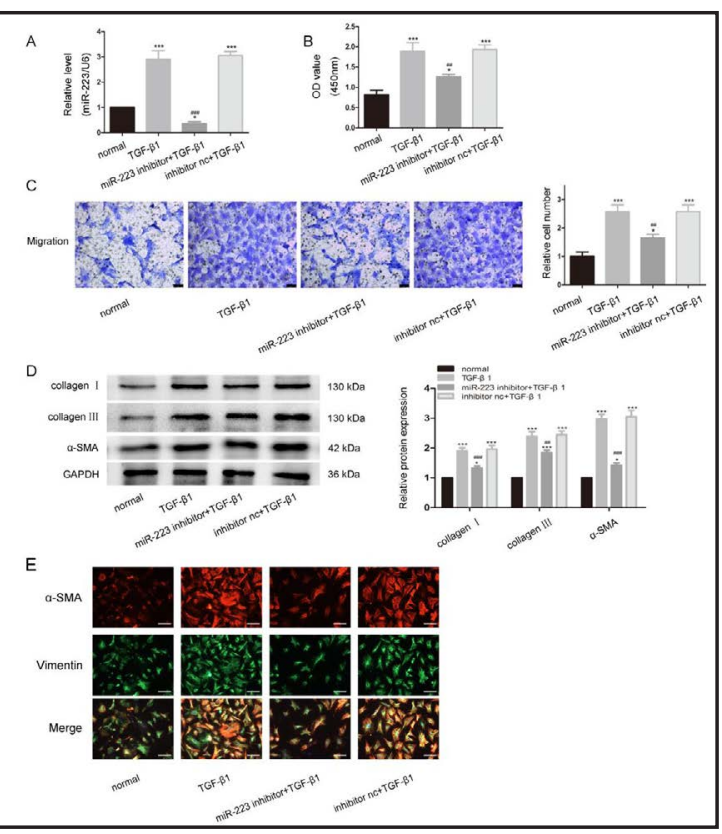
collagen III, and $\alpha$-SMA expression after treating the transfected CFs with TGF- $\beta 1(10 \mathrm{ng} / \mathrm{ml}$ ) for 48 hours, $\mathrm{n}=3$. (E) Immunofluorescence images showing the fluorescence intensity after treating the transfected CFs with TGF- $\beta 1(10 \mathrm{ng} / \mathrm{ml})$ for 48 hours. a-SMA was detected by immunofluorescence using anti-a-SMA antibody, Cy3-conjugated goat anti-rabbit secondary antibody (red staining). Vimentin was detected by immunofluorescence using anti-vimentin antibody, FITCconjugated goat anti-mouse secondary antibody (green staining), and DAPI for nuclei (blue). Undur 200x

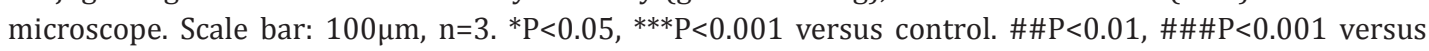
TGF-ß1.

Fig. 4. RASA1 is a directtarget of miR-223 in CFs. (A)(B) qRT-PCR analysis of RASA1 mRNA expression levels in CFs transfected with miR-223 mimic, miR-223 inhibitor, or the corresponding negative controls for 24 hours. ${ }^{*} \mathrm{P}<0.05$ versus mimic nc, $\# \mathrm{P}<0.05$ versus inhibitor $\mathrm{nc}, \mathrm{n}=3$. (C)(D) Representative western blotting results showing RASA1 protein expression levels in CFs transfected with miR-223 mimic, miR-223 inhibitor, or the corresponding negative controls for 48 hours, $\mathrm{n}=3$. (E) The predicted binding sites for miR-223 in the WT and MUT 3'UTRs of RASA1. (F) Dual-luciferase reporter assay showing the relative luciferase activity in CFs co-transfected with either RASA1 3'UTR WT plasmid or RASA1 3'UTR MUT plasmid and miR-223 mimic or mimic $\mathrm{nc}, \mathrm{n}=3 . * * \mathrm{P}<0.01$ versus RASA1 3'UTR WT+mimic nc.

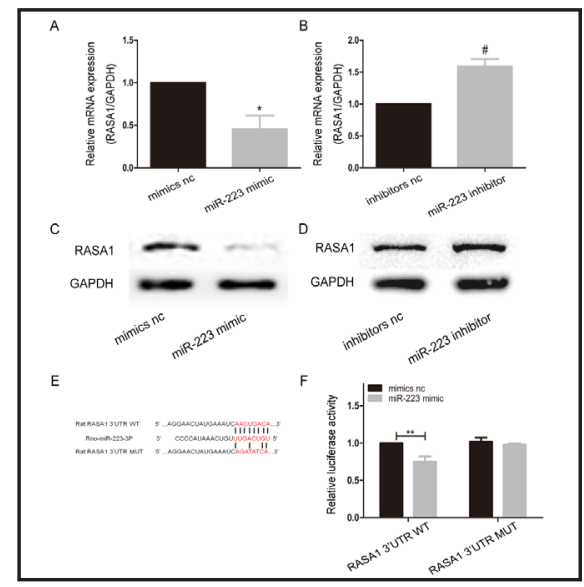

downregulated RASA1 mRNA and protein expression levels in CFs; however, the miR-223 inhibitor had contrasting effects (Fig. 4A, B, C, D). Moreover, dual-luciferase reporter assay showed that the luciferase activity of the WT 3'UTR of RASA1 was significantly repressed in the miR-223 mimic group compared with the normal control group (Fig. 4F). These results demonstrate that miR-223 directly targets RASA1 in CFs.

\section{RASA1 is involved in the process by which CFs are regulated by miR-223 in vitro}

We showed that miR-223 directly targets RASA1 in CFs; however, whether RASA1 participates in the process by which miR-223 regulates CFs activation remains unknown. Therefore, we transfected CFs with siRASA1-with or without a miR-223 inhibitor-to 
knock down RASA1. siRASA1 inhibited RASA1 mRNA and protein expressions (Fig. 5A), and depressed RASA1 protein expression followed by miR-223 inhibitor transfection as well (Fig. 6D), indicating high transfection efficiency in both cases. Moreover, siRASA1 enhanced CFs proliferation and migration, as demonstrated by the CCK8 and transwell migration and scratch assays (Fig. 5B, C, D). Most importantly, RASA1 knockdown reversed the inhibitory effects of the miR-223 inhibitor on CFs proliferation and migration ability (Fig. 6A, B, C). Western blotting revealed that siRASA1 promoted collagen I, collagen III and $\alpha$-SMA expression and reversed the inhibitory effects of the miR-223 inhibitor on the expression of these proteins (Fig. 5E, Fig. 6D). Moreover, immunofluorescence analysis of $\alpha$-SMA expression (Fig. 5F, Fig. 6E) confirmed that siRASA1 regulates CFs differentiation; thus, siRASA1 promotes not only cell proliferation and migration but also myofibroblast differentiation. These results indicated that the knockdown of RASA1 in CFs phenocopies miR-223 overexpression, suggesting that RASA1 has an epistatic relationship with miR-223 and that the protein mediates the effects of miR-223 on CFs.

miR-223 inhibition in vivo attenuates cardiac fibrosis and improves ventricular function post-MI

As demonstrated above, miR-223 plays vital roles in CFs. Because miR-223 downregulation impedes CFs proliferation, migration, and myofibroblast differentiation, we speculated that the extensive cardiac fibrosis and cardiac functional impairments that develop after MI can be improved by miR-223 knockdown. We investigated the effects of miR-223 knockdown on cardiac structure and function by inhibiting miR-223 in vivo with a cardiotropic AAV9 delivery system. Using qRT-PCR, we found that miR-223 expression was remarkably increased by more than 7 fold in the MI group compared with the control group. Moreover, we found that the miR-223 sponge AAV9 efficiently prevented MI-induced enhancements of miR-223 expression (Fig. 7B). As shown in Fig. 7A, miR-223 knockdown

Fig. 5. RASA1 is involved in the process by which CFs are regulated by miR-223 in vitro. Knockdown of RASA1 with siRASA1 could promote CFs proliferation, migration, and myofibroblast differentiation in vitro. CFs were transfected with siRASA1 or siNC for appointed times and then subjected to the following assays. (A) qRT-PCR analysis of RASA1 mRNA expression levels in CFs after transfection for 24 hours, $n=3$. (B) CCK8 assay showing CFs proliferation at the different times $(24,48,72,96$ hours) after transfection, $n=3$. (C) Transwell assay showing CFs migration ability. After transfection for 48 hours, CFs were digested with trypsin and then $50000 \mathrm{CFs}$ in siRASA1 or siNC group were plated in the upper chambers of tranwell culture plate. After 24 hours, the migrated CFs were dyed and culpture under 100x microscope. Scale bar: $100 \mu \mathrm{m}, \mathrm{n}=3$. (D) scratch assays showing CFs migration ability after transfection for 48 hours, 100x, scale bar: $100 \mu \mathrm{m}$, $n=3$. (E) Representative western blotting results and quantitative data pertaining to collagen I, collagen

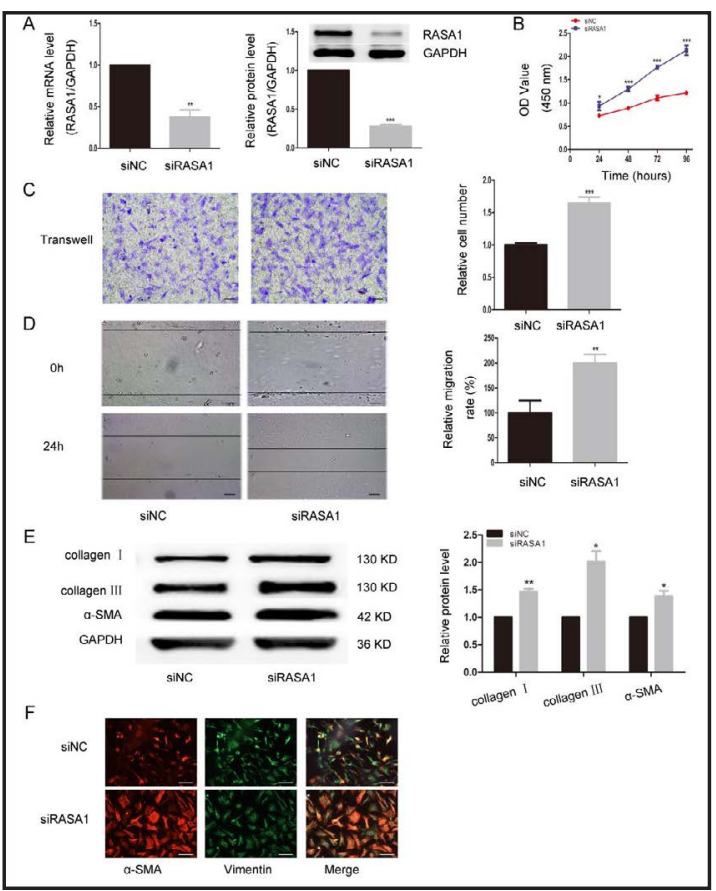
III, and $\alpha$-SMA expression after transfection for 48 hours, n=3. (F) Immunofluorescence images showing the fluorescence intensity after transfection for 48 hours. a-SMA was detected by immunofluorescence using anti-a-SMA antibody, Cy3-conjugated goat anti-rabbit secondary antibody (red staining). Vimentin was detected by immunofluorescence using antivimentin antibody, FITC-conjugated goat anti-mouse secondary antibody (green staining), and DAPI for nuclei (blue). Undur 200x microscope. Scale bar: $100 \mu \mathrm{m}, \mathrm{n}=3$. ${ }^{*} \mathrm{P}<0.05,{ }^{* *} \mathrm{P}<0.01,{ }^{* * *} \mathrm{P}<0.001$ versus si NC. 
Fig. 6. RASA1 is involved in the process by which CFs are regulated by miR-223 in vitro. Knockdown of RASA1 with siRASA1 in CFs before miR-223 inhibitors transfection could reverse the inhibitory effects of miR-223 inhibitors on CFs proliferation, migration, and myofibroblast differentiation in vitro. (A) CFs were transfected with siRASA1 or siNC for 24 hours and then subjected to miR-223 inhibitors or inhibitors nc transfection for different times $(24,48,72,96$ hours) for CCK8 assays to determine the proliferation of CFs, $n=3$. (B) CFs were transfected with siRASA1 or siNC for 24 hours, and then transfected with miR-223 inhibitors or inhibitors nc for 48 hours. Then the transfected CFs were digested with trypsin, and 50000 CFs in each group were plated in the upper chambers of tranwell culture plate. After 24 hours, the migrated CFs were dyed and culpture under

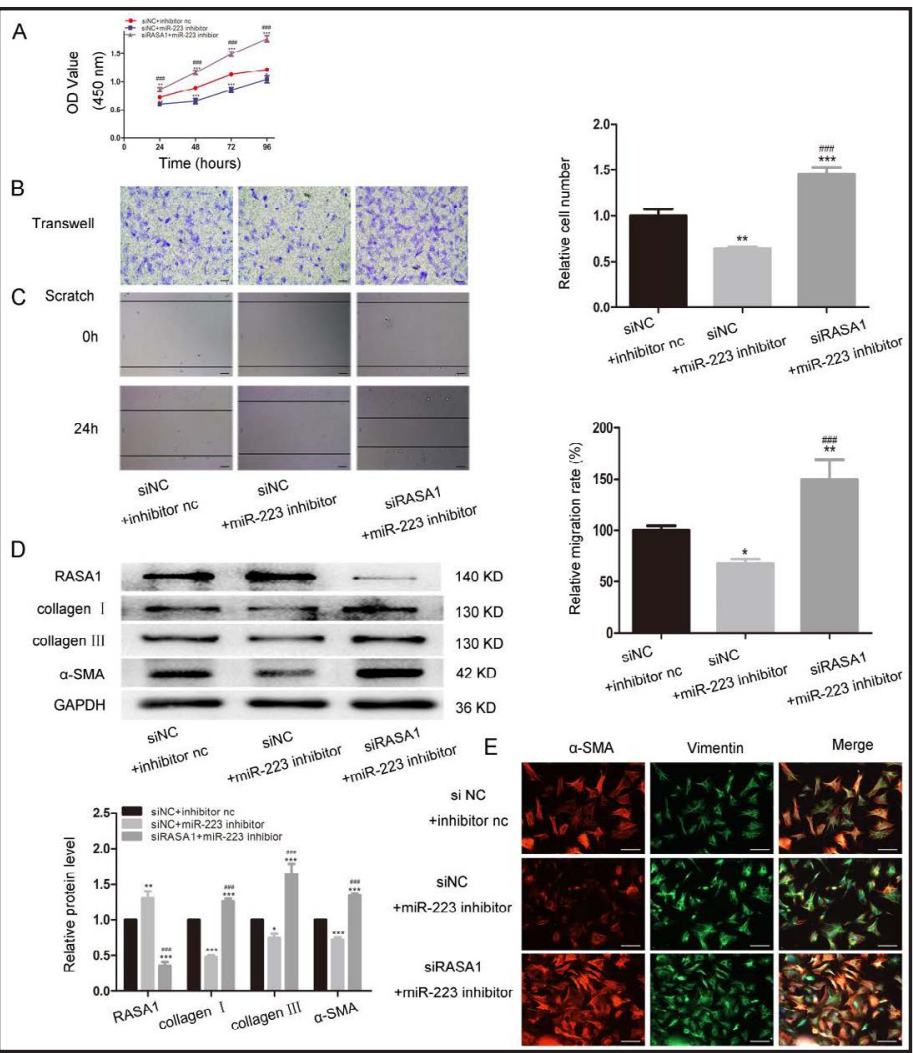
100x microscope. Scale bar: $100 \mu \mathrm{m}, \mathrm{n}=3$. (C) CFs were transfected with siRASA1 or siNC for 24 hours and then transfected with miR-223 inhibitors or inhibitors nc transfection for 48 hours for scratch assays to determine the migration of CFs, 100x, scale bar: $100 \mu \mathrm{m}, \mathrm{n}=3$. (D)(E) Representative western blotting results and quantitative data pertaining to RASA1, collagen I, collagen III, $\alpha$-SMA, and immunofluorescence images showing the fluorescence intensity in CFs transfected with siRASA1 or siNC for 24 hours and then transfected with miR-223 inhibitors or inhibitors nc transfection for 48 hours, $n=3$. a-SMA was detected by immunofluorescence using anti-a-SMA antibody, Cy3-conjugated goat anti-rabbit secondary antibody (red staining). Vimentin was detected by immunofluorescence using anti-vimentin antibody, FITC-conjugated goat anti-mouse secondary antibody (green staining), and DAPI for nuclei (blue). Under 200x microscope. Scale bar: $100 \mu \mathrm{m}, \mathrm{n}=3$. ${ }^{*} \mathrm{P}<0.05,{ }^{* *} \mathrm{P}<0.01,{ }^{* *} \mathrm{P}<0.001$ versus siNC+inhibitor $\mathrm{nc}, \# \mathrm{P}<0.05, \# \# \mathrm{P}<0.01$, \#\#\#P<0.001 versus siNC+miR-223 inhibitor.

reduced post-AMI heart volumes, suggesting that miR-223 inhibition alleviated cardiac remodeling. Furthermore, the echocardiography data showed that AAV9-mediated miR-223 inhibition significantly preserved the left ventricular ejection fraction (EF) and fractional shortening (FS) in rats at 8 weeks post-MI (Fig. 7C). However, there were no significant differences in miR-223 expression, cardiac function, and heart volume between the MI and MI+miR-scramble groups, indicating that the AAV9 vector did not impact the animals themselves. Inhibiting miR-223 in the heart reduced collagen deposition in the border zones of infarcted myocardial tissues, as demonstrated by Masson's trichrome staining (Fig. 7D). Moreover, post-MI collagen I and collagen III expression levels in the ventricle were decreased by miR-223 inhibition, as were $\alpha$-SMA expression levels (Fig. 7E). These data serve as strong evidence showing that miR-223 inhibition has cardioprotective effects against cardiac fibrosis and thus improves cardiac function post-MI.

MiR-223 regulates various signaling pathways both in vivo and in vitro

RAS proteins signal through multiple effector pathways, including the MEK/ERK (MAPK) and PI3K/AKT pathways, which have been investigated extensively and are related 
Fig. 7. MiR-223 inhibition in vivo attenuates cardiac fibrosis and improves ventricular function post-MI. The LAD coronary artery of rats were ligated to build up MI models. To downregulate the expression of miR-223 in MI models, miR-223 sponge or miR-scramble mediated by AAV9 were injected intramyocardially before LAD ligation. After 8 weeks, the rats were sacrificed and the following assays were conducted. (A) Appearances of the hearts in each group. (B) qRT-PCR analysis of miR-223 expression in rat myocardial tissues, $n=3$. (C) Functional and quantitative echocardiographic analysis of the left ventricle. The EF and FS are shown, $n=6$. (D) Masson's trichrome staining of rat heart tissue sections showing interstitial fibrosis in the border zones of the infarcted regions, $n=6$. (E) Representative western blotting results and quantitative data, $\mathrm{n}=6$.

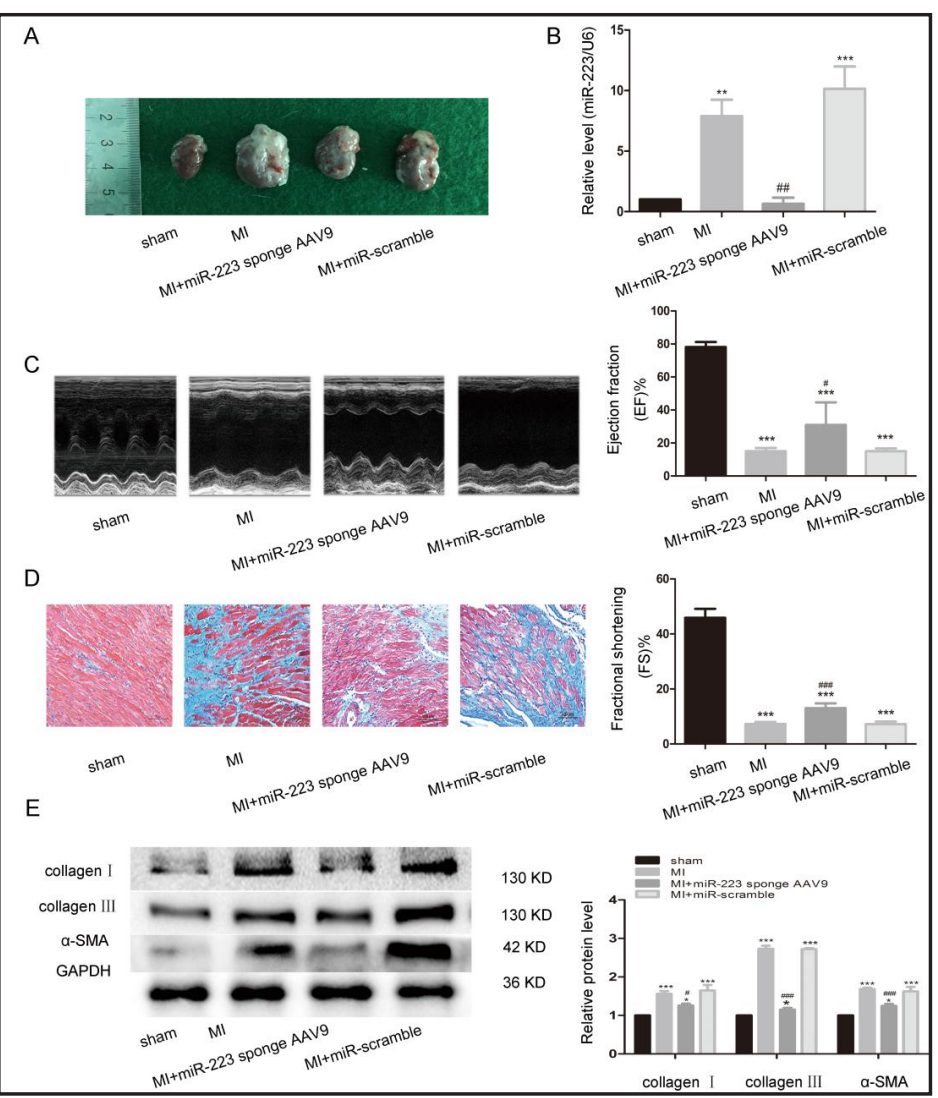

${ }^{*} \mathrm{P}<0.05,{ }^{* *} \mathrm{P}<0.01,{ }^{* * *} \mathrm{P}<0.001$ versus sham, $\# \mathrm{P}<0.05$, \#\#P<0.05, \#\#\#P<0.001 versus MI.

Fig. 8. MiR-223 regulates various signaling pathways both in vitro and in vivo. The phosphorylation levels of MEK1/2,ERK1/2 and AKT were shown by representative western blots and quantitative analysis under various treatments in vitro and in vivo. (A) The cultured CFs were transfected with miR-223 mimic or inhibitor or their corresponding negative controls for 48 hours in vitro. $\mathrm{N}=3, * \mathrm{P}<0.05,{ }^{*} \mathrm{P}<0.01$, $* * * \mathrm{P}<0.001$ versus mimic $\mathrm{nc}, \# \mathrm{P}<0.05$, $\# \# \mathrm{P}<0.01$ versus inhibitor nc. (B) The cultured CFs were transfected with SiRASA 1 or siNC for 48 hours or transfected with siRASA1 or siNC for 24 hours and then transfected with miR-223 inhibitor or inhibitor nc for 48 hours in vitro. $\$ \mathrm{P}<0.05, \quad \$ \$ \mathrm{P}<0.01, \quad \$ \$ \mathrm{P}<0.001$ versus siNC. $\& \mathrm{P}<0.05, \quad \& \& \mathrm{P}<0.01, \quad \& \& \& \mathrm{P}<0.001$ versus siNC+inhibitor nc, $\% \% \mathrm{P}<0.01$, $\% \% \% \mathrm{P}<0.001$ versus siNC+miR-223 inhibitor. $\mathrm{N}=3$. (C) The in vivo models were constructed and protein expression of RASA1 and phosphorylation levels of MEK1/2, ERK1/2 and AKT were detected. $\mathrm{N}=6 .{ }^{* *} \mathrm{P}<0.01,{ }^{* * *} \mathrm{P}<0.001$ versus sham, $\# \mathrm{P}<0.05$, \#\#P<0.01, \#\#\#P<0.001 versus $\mathrm{MI}$.

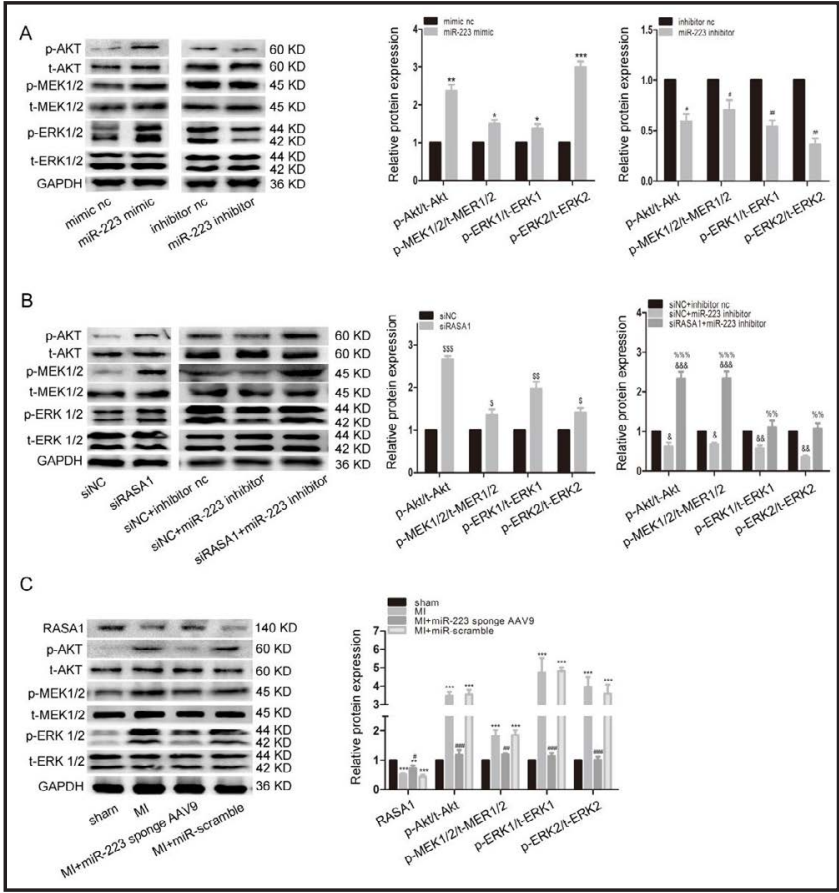




\section{Cellular Physiology Cell Physiol Biochem 2018;46:1439-1454

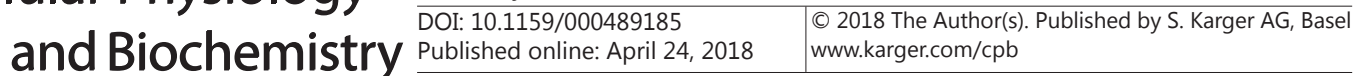

Liu et al.: The Roles of MicroRNA-223 in Cardiac Fibrosis

to various cardiac diseases $[24,25]$. This study showed that RASA1 was responsible for the effects of miR-223 in CFs. Therefore, we analyzed the changes in the protein expression of the major downstream effectors of the RAS signaling pathway, namely, MEK1/2 and p-MEK1/2, ERK1/2 and pERK1/2, and AKT and p-AKT, in CFs in which miR-223 was upor downregulated or RASA1 was downregulated and in the in vivo rat model. As shown in Fig. 8A and B, miR-223 overexpression and RASA1 downregulation promoted MEK1/2, ERK1/2, and AKT phosphorylation in CFs, while miR-223 inhibition had contrasting effects (Fig. 8A). Moreover, siRASA1 reversed the reductions in protein phosphorylation induced by the miR-223 inhibitor (Fig. 8B). Furthermore, MI increased p-MEK1/2, P-ERK1/2, and P-AKT expression, while AAV9-mediated miR-223 inhibition prevented MI-induced increases in the expression of the above proteins (Fig. 8C). These findings suggested that miR-223 downregulated RASA1 expression, thereby activating the RAS signaling pathways, and therefore played an important role in promoting CFs proliferation, migration and differentiation.

\section{Discussion}

Post-MI cardiac fibrosis has been shown to be a key factor in heart failure development [26]. Long-term post-MI cardiac remodeling leads to the deposition of fibrous material in the myocardium, a change that impairs systolic function and thus aggravates heart failure. Therefore, increasing numbers of studies have suggested that anti-fibrotic therapies may be an effective approach to treating heart failure [27]. miRNAs, small non-coding RNAs, have been widely studied and participate in various physiological and pathological processes. Accumulating evidence indicates that the aberrant expression of some miRNAs and the processes in which these miRNAs participate play crucial roles in cardiac fibrosis and heart failure [28]. In the present study, for the first time, we determined the roles of miR223 in cardiac fibrosis and elucidated the mechanisms underlying its effects. Our in vitro experiments showed that miR-223 overexpression promoted CFs proliferation, migration, and differentiation, while miR-223 downregulation had contrasting effects and mediated the effects of TGF- $\beta 1$. These findings indicate that miR-223 is a pro-fibrotic factor. Moreover, we presented strong evidence showing that RASA1 is a target gene of miR-223 and is responsible for the effects of miR-223 in CFs. Subsequent in vivo experiments in which miR223 expression was downregulated by the miR-223 sponge AAV9 showed that miR-223 inhibition alleviated cardiac fibrosis and improved cardiac function. Therefore, miR-223 is a critical pro-fibrotic factor and may be a new target in the treatment of cardiac disease.

MiR-223 has been implicated in numerous physiological and pathological processes [29-31], and several studies have reported that miR-223 is associated with myocardial diseases. Lu et al. reported that miR-223 was upregulated in left ventricular biopsies from patients with type 2 diabetes and that overexpressing miR-223 increased glucose uptake by upregulating Glut4 protein expression [32]. Wang et al. showed that miR-223 was downregulated in ET-1 induced hypertrophic CMs and hypertrophic myocardium and that the miRNA alleviates cardiomyocyte hypertrophy by targeting TNNI3K [33]. However, Wang et al. reported that miR-223 promotes cardiac hypertrophy, in which its expression is elevated [34]. The contrasting findings regarding the role of miR-223 in hypertrophic myocardial tissue may be attributable to differences in the stimuli used in the hypertrophic models in the indicated studies, as well as between-study differences in the miR-223 target genes under investigation. The effects of miR-223 in hearts subjected to ischemia/reperfusion (I/R) have also been investigated. Both strands (5p and 3p) of miR-223 were remarkably dysregulated in mouse hearts subjected to $I / R$, in which they cooperatively suppressed necroptosis [35]. Liu et al. reported that miR-223 was upregulated in a rat model of AMI and that its overexpression can promote arrhythmias; thus, miR-223 may be a new target in the treatment of ischemic arrhythmias [36]. The abovementioned studies elucidated the roles of miR-223 in various cardiac diseases, focusing specifically on the roles of miR-223 in various 
metabolic, hypertrophic and necrotic processes; however, they did not investigate whether or not miR-223 impacts cardiac fibrosis. One study reported that miR-223 was aberrantly expressed in the myocardium in patients with post-MI heart failure [7]. and another study found that miR-223 expression is altered in rat peritoneal fibrosis [8]. However, these studies did not investigate the effects of miR-223 further. Therefore, we thought that it would be important to examine whether alterations in miR-223 expression impact the pathogenesis of post-infarction heart failure. In particular, we paid close attention to the effects of miR-223 on cardiac fibrosis. We demonstrated that miR-223 is a pro-fibrotic factor in MI and may be a target in the treatment of MI-induced heart failure.

RASA1 has been reported to participate in various processes, including anti- and pro-apoptosis and cell proliferation and migration in angiogenesis and cancer [37]. More importantly, several studies have noted that RASA1 is associated with cardiac diseases. One study showed that RASA1 plays a role in cardiomyocyte growth induced by hypertrophic stimuli [38], and another study predicted that RASA1 is one of the target genes of miR1. An increase in RASA1 mRNA expression was observed in hypertrophic diabetic hearts [39] . However, no investigations have determined whether or not RASA is related to the pathogenesis of post-MI heart failure. Moreover, no studies have determined whether or not RASA1 participates in fibrosis. Our study confirmed that RASA1 is a target gene of miR-223. Moreover, we showed that similar to miR-223 overexpression, RASA1 knockdown promoted CFs proliferation, migration, and differentiation and reversed the inhibitory effects of miR-223 inhibition on CFs proliferation, migration, and differentiation. However, miR-223 inhibition in vivo improved cardiac function and upregulated RASA1 expression post-MI. Thus, we assumed that miR-223 impacts cardiac fibrosis in part by targeting RASA1. The RAS/ MEK/ERK and RAS/PI3K/AKT pathways are two important RAS protein effector pathways that participate in a wide range of cellular processes, including cell growth, proliferation, differentiation, and survival. Previous studies have demonstrated that the MEK/ERK and PI3K/AKT pathways are closely associated with cardiac fibrosis [40-42]. Thus, we elected to investigate whether miR-223 and RASA1 impact the MEK/ERK and PI3K/AKT pathways. We found that miR-223 overexpression and RASA1 knockdown induced increases in p-MEK1/2, p-ERK1/2, and p-AKT expression in CFs, while miR-223 downregulation had contrasting effects. Moreover, miR-223 inhibition in vivo attenuated MI-induced enhancements of p-MEK1/2, p-ERK1/2, and p-AKT expression.

TGF- $\beta 1$ is considered the most potent profibrogenic cytokine. Apart from the canonical Smad signaling pathway, TGF- $\beta 1$ also activates various Smad-independent signaling pathways, including PI3K/AKT [43-45] and ERK 1/2 [46]. In the current study, we revealed that TGF- $\beta 1$ increased the expression of miR-223 and miR-223 partially mediated the effects of TGF- $\beta 1$ on CFs proliferation, migration and differentiation. Moreover, miR-223 could promote the activation of RAS signaling patwhay through targeting RASA1. Thus, we speculate that miR-223 mediated the effects of TGF- $\beta 1$ on these two kinds of RAS signaling pathway as well. Several studies have revealed that Ras protein and ERKs could mediate the effects of TGF- $\beta 1$ on the promoters of genes that are involved in TGF- $\beta$-stimulated accumulation of extracellular matrix $[46,47]$, and were critical for the enhancement of DNA synthesis in CFs [48]. Besides, the PI3K/Akt pathway is involved in the regulation of TGF- $\beta 1$ mediated transcription [43]. Thus, we assume that in CFs, TGF- $\beta 1$ stimulates the expression of miR-223 and the latter negatively regulates RASA1, then activating RAS signaling pathways, and in return RAS signaling pathways acts synergistically with the Smad signaling pathway activated by TGF- $\beta 1$, resulting in the activation of CFs and increased synthesis of extracellular matrix eventually. However, we did not investigate whether the pro-fibrotic effects of miR-223 and RASA1 are mediated by the MEK/ERK and PI3K/AKT pathways, nor did we determine whether blocking the pathways can reverse the effects. Additionally, we did not determine the extent to which the two pathways contribute to the pro-fibrotic effects of miR-223 and RASA1, and the interaction between RAS signaling pathways and TGF- $\beta 1$ in CFs is still undetermined, which remain to be clarified further. 
In conclusion, our study investigated the impact of miR-223 on cardiac fibrosis for the first time. We found that miR-223 is a potent pro-fibrotic factor and that inhibiting miR-223 expression in vivo in a post-MI heart failure model alleviated cardiac fibrosis and significantly improved cardiac function. Furthermore, we found that RASA1 is a target gene of miR-223 in the pathogenesis of fibrosis. RASA1 is a RAS signaling pathway-related protein. We also found that miR-223 advanced cardiac fibrosis in part by targeting RASA1; therefore, miR223 regulates RAS signaling pathways, such as the MEK/ERK and PI3K/AKT pathways. Collectively, our findings indicate that miR-223 promotes cardiac fibrosis; therefore, miR223 inhibition may be a strategy for treating cardiac fibrosis.

\section{Acknowledgements}

This work was supported by funding from Shanghai General Hospital (0601N16094).

\section{Disclosure Statement}

No conflict of interests exists.

\section{References}

1 Zamilpa R, Lindsey ML: Extracellular matrix turnover and signaling during cardiac remodeling following MI: causes and consequences. J Mol Cell Cardiol 2010;48:558-563.

-2 Tomasek JJ, Gabbiani G, Hinz B, Chaponnier C, Brown RA: Myofibroblasts and mechano-regulation of connective tissue remodelling. Nat Rev Mol Cell Biol 2002;3:349-363.

-3 van den Borne SW, Diez J, Blankesteijn WM, Verjans J, Hofstra L, Narula J: Myocardial remodeling after infarction: the role of myofibroblasts. Nat Rev Cardiol 2010;7:30-37.

-4 Olson EN: MicroRNAs as therapeutic targets and biomarkers of cardiovascular disease. Sci Transl Med 2014;6:239ps233. Abdellatif M: Differential expression of microRNAs in different disease states. Circ Res 2012;110:638-650. Yang B, Lu Y, Wang Z: Control of cardiac excitability by microRNAs. Cardiovasc Res 2008;79:571-580. van Rooij E, Sutherland LB, Thatcher JE, DiMaio JM, Naseem RH, Marshall WS, Hill JA, Olson EN: Dysregulation of microRNAs after myocardial infarction reveals a role of miR-29 in cardiac fibrosis. Proc Natl Acad Sci U S A 2008;105:13027-13032.

8 Morishita Y, Yoshizawa H, Watanabe M, Imai R, Imai T, Hirahara I, Akimoto T, Ookawara S, Muto S, Nagata D: MicroRNA expression profiling in peritoneal fibrosis. Transl Res 2016;169:47-66.

-9 Proud CG: Ras, PI3-kinase and mTOR signaling in cardiac hypertrophy. Cardiovasc Res 2004;63:403-413.

10 Sugden PH: Ras, Akt, and mechanotransduction in the cardiac myocyte. Circ Res 2003;93:1179-1192.

11 Wittinghofer A: Signal transduction via Ras. Biol Chem 1998;379:933-937.

12 Trial J, Entman ML, Cieslik KA: Mesenchymal stem cell-derived inflammatory fibroblasts mediate interstitial fibrosis in the aging heart. J Mol Cell Cardiol 2016;91:28-34.

13 Tao H, Yang JJ, Chen ZW, Xu SS, Zhou X, Zhan HY, Shi KH: DNMT3A silencing RASSF1A promotes cardiac fibrosis through upregulation of ERK1/2. Toxicology 2014;323:42-50.

14 Li L, Fan D, Wang C, Wang JY, Cui XB, Wu D, Zhou Y, Wu LL: Angiotensin II increases periostin expression via Ras/p38 MAPK/CREB and ERK1/2/TGF-beta1 pathways in cardiac fibroblasts. Cardiovasc Res 2011;91:8089.

15 Queiros AM, Eschen C, Fliegner D, Kararigas G, Dworatzek E, Westphal C, Sanchez Ruderisch H, RegitzZagrosek V: Sex- and estrogen-dependent regulation of a miRNA network in the healthy and hypertrophied heart. Int J Cardiol 2013;169:331-338.

16 Li X, Li D, Wikstrom JD, Pivarcsi A, Sonkoly E, Stahle M, Landen NX: MicroRNA-132 promotes fibroblast migration via regulating RAS p21 protein activator 1 in skin wound healing. Sci Rep 2017;7:7797.

-17 Ying W, Tseng A, Chang RC, Morin A, Brehm T, Triff K, Nair V, Zhuang G, Song H, Kanameni S, Wang H, 


\section{Cellular Physiology Cell Physiol Biochem 2018;46:1439-1454 \begin{tabular}{ll|l} 
and Biochemistry Published online: April 24, 2018 & $\begin{array}{l}\text { C) } 2018 \text { The Author(s). Published by S. Karger AG, Basel } \\
\text { www.karger.com/cpb }\end{array}$ \\
\hline
\end{tabular}}

Liu et al.: The Roles of MicroRNA-223 in Cardiac Fibrosis

Golding MC, Bazer FW, Chapkin RS, Safe S, Zhou B: MicroRNA-223 is a crucial mediator of PPARgammaregulated alternative macrophage activation. J Clin Invest 2015;125:4149-4159.

18 Sun D, Wang C, Long S, Ma Y, Guo Y, Huang Z, Chen X, Zhang C, Chen J, Zhang J: C/EBP-beta-activated microRNA-223 promotes tumour growth through targeting RASA1 in human colorectal cancer. Br J Cancer 2015;112:1491-1500.

19 Gan XT, Ettinger G, Huang CX, Burton JP, Haist JV, Rajapurohitam V, Sidaway JE, Martin G, Gloor GB, Swann JR, Reid G, Karmazyn M: Probiotic administration attenuates myocardial hypertrophy and heart failure after myocardial infarction in the rat. Circ Heart Fail 2014;7:491-499.

20 Aoyama Y, Kobayashi K, Morishita Y, Maeda K, Murohara T: Wnt11 gene therapy with adeno-associated virus 9 improves the survival of mice with myocarditis induced by coxsackievirus B3 through the suppression of the inflammatory reaction. J Mol Cell Cardiol 2015;84:45-51.

21 Karakikes I, Chaanine AH, Kang S, Mukete BN, Jeong D, Zhang S, Hajjar RJ, Lebeche D: Therapeutic cardiactargeted delivery of miR-1 reverses pressure overload-induced cardiac hypertrophy and attenuates pathological remodeling. J Am Heart Assoc 2013;2:e000078.

22 Tao L, Bei Y, Chen P, Lei Z, Fu S, Zhang H, Xu J, Che L, Chen X, Sluijter JP, Das S, Cretoiu D, Xu B, Zhong J, Xiao J, Li X: Crucial Role of miR-433 in Regulating Cardiac Fibrosis. Theranostics 2016;6:2068-2083.

23 Nishida M, Onohara N, Sato Y, Suda R, Ogushi M, Tanabe S, Inoue R, Mori Y, Kurose H: Galpha12/13mediated up-regulation of TRPC6 negatively regulates endothelin-1-induced cardiac myofibroblast formation and collagen synthesis through nuclear factor of activated T cells activation. J Biol Chem 2007;282:23117-23128.

24 Muslin AJ: MAPK signalling in cardiovascular health and disease: molecular mechanisms and therapeutic targets. Clin Sci (Lond) 2008;115:203-218.

-25 Xu Z, Sun J, Tong Q Lin Q Qian L, Park Y, Zheng Y: The Role of ERK1/2 in the Development of Diabetic Cardiomyopathy. Int J Mol Sci 2016;17:

-26 Porter KE, Turner NA: Cardiac fibroblasts: at the heart of myocardial remodeling. Pharmacol Ther 2009;123:255-278.

-27 Segura AM, Frazier OH, Buja LM: Fibrosis and heart failure. Heart Fail Rev 2014;19:173-185. Wang H, Cai J: The role of microRNAs in heart failure. Biochim Biophys Acta 2017;1863:2019-2030. Kurozumi A, Goto Y, Matsushita R, Fukumoto I, Kato M, Nishikawa R, Sakamoto S, Enokida H, Nakagawa M, Ichikawa T, Seki N: Tumor-suppressive microRNA-223 inhibits cancer cell migration and invasion by targeting ITGA3/ITGB1 signaling in prostate cancer. Cancer Sci 2016;107:84-94.

-30 Li J, Guo Y, Liang X, Sun M, Wang G, De W, Wu W: MicroRNA-223 functions as an oncogene in human gastric cancer by targeting FBXW7/hCdc4. J Cancer Res Clin Oncol 2012;138:763-774.

-31 Zhang J, Luo X, Li H, Yue X, Deng L, Cui Y, Lu Y: MicroRNA-223 functions as an oncogene in human colorectal cancer cells. Oncol Rep 2014;32:115-120.

-32 Lu H, Buchan RJ, Cook SA: MicroRNA-223 regulates Glut4 expression and cardiomyocyte glucose metabolism. Cardiovasc Res 2010;86:410-420.

-33 Wang YS, Zhou J, Hong K, Cheng XS, Li YG: MicroRNA-223 displays a protective role against cardiomyocyte hypertrophy by targeting cardiac troponin I-interacting kinase. Cell Physiol Biochem 2015;35:1546-1556.

-34 Wang K, Long B, Liu F, Wang JX, Liu CY, Zhao B, Zhou LY, Sun T, Wang M, Yu T, Gong Y, Liu J, Dong YH, Li N, Li PF: A circular RNA protects the heart from pathological hypertrophy and heart failure by targeting miR223. Eur Heart J 2016;37:2602-2611.

35 Qin D, Wang X, Li Y, Yang L, Wang R, Peng J, Essandoh K, Mu X, Peng T, Han Q Yu KJ, Fan GC: MicroRNA223-5p and -3p Cooperatively Suppress Necroptosis in Ischemic/Reperfused Hearts. J Biol Chem 2016;291:20247-20259.

-36 Liu X, Zhang Y, Du W, Liang H, He H, Zhang L, Pan Z, Li X, Xu C, Zhou Y, Wang L, Qian M, Liu T, Yin H, Lu Y, Yang B, Shan H: MiR-223-3p as a Novel MicroRNA Regulator of Expression of Voltage-Gated K+ Channel Kv4.2 in Acute Myocardial Infarction. Cell Physiol Biochem 2016;39:102-114.

37 Pamonsinlapatham P, Hadj-Slimane R, Lepelletier Y, Allain B, Toccafondi M, Garbay C, Raynaud F: p120Ras GTPase activating protein (RasGAP): a multi-interacting protein in downstream signaling. Biochimie 2009;91:320-328.

-38 Lypowy J, Chen IY, Abdellatif M: An alliance between Ras GTPase-activating protein, filamin C, and Ras GTPase-activating protein SH3 domain-binding protein regulates myocyte growth. J Biol Chem 2005;280:25717-25728. 


\section{Cellular Physiology Cell Physiol Biochem 2018;46:1439-1454 \begin{tabular}{l|l} 
DOI: 10.1159/000489185 & and Biochemistry \\
Published 2018 The Author(s). Published by S. Karger AG, Basel \\
www.karger.com/cpb
\end{tabular}}

Liu et al.: The Roles of MicroRNA-223 in Cardiac Fibrosis

-39 Diao X, Shen E, Wang X, Hu B: Differentially expressed microRNAs and their target genes in the hearts of streptozotocin-induced diabetic mice. Mol Med Rep 2011;4:633-640.

40 Phosri S, Arieyawong A, Bunrukchai K, Parichatikanond W, Nishimura A, Nishida M, Mangmool S: Stimulation of Adenosine A2B Receptor Inhibits Endothelin-1-Induced Cardiac Fibroblast Proliferation and alpha-Smooth Muscle Actin Synthesis Through the cAMP/Epac/PI3K/Akt-Signaling Pathway. Front Pharmacol 2017;8:428.

41 Lin CS, Pan CH: Regulatory mechanisms of atrial fibrotic remodeling in atrial fibrillation. Cell Mol Life Sci 2008;65:1489-1508.

-42 Li X, Zhang ZL, Wang HF: Fusaric acid (FA) protects heart failure induced by isoproterenol (ISP) in mice through fibrosis prevention via TGF-beta1/SMADs and PI3K/AKT signaling pathways. Biomed Pharmacother 2017;93:130-145.

43 Bakin AV, Tomlinson AK, Bhowmick NA, Moses HL, Arteaga CL: Phosphatidylinositol 3-kinase function is required for transforming growth factor beta-mediated epithelial to mesenchymal transition and cell migration. J Biol Chem 2000;275:36803-36810.

44 Yi JY, Shin I, Arteaga CL: Type I transforming growth factor beta receptor binds to and activates phosphatidylinositol 3-kinase. J Biol Chem 2005;280:10870-10876.

45 Wilkes MC, Mitchell H, Penheiter SG, Dore JJ, Suzuki K, Edens M, Sharma DK, Pagano RE, Leof EB: Transforming growth factor-beta activation of phosphatidylinositol 3-kinase is independent of Smad 2 and Smad3 and regulates fibroblast responses via p21-activated kinase-2. Cancer Res 2005;65:10431-10440.

46 Mucsi I, Skorecki KL, Goldberg HJ: Extracellular signal-regulated kinase and the small GTP-binding protein, Rac, contribute to the effects of transforming growth factor-beta1 on gene expression. J Biol Chem 1996;271:16567-16572.

-47 Stratton R, Rajkumar V, Ponticos M, Nichols B, Shiwen X, Black CM, Abraham DJ, Leask A: Prostacyclin derivatives prevent the fibrotic response to TGF-beta by inhibiting the Ras/MEK/ERK pathway. FASEB J 2002;16:1949-1951.

48 Zou Y, Komuro I, Yamazaki T, Kudoh S, Aikawa R, Zhu W, Shiojima I, Hiroi Y, Tobe K, Kadowaki T, Yazaki Y: Cell type-specific angiotensin II-evoked signal transduction pathways: critical roles of Gbetagamma subunit, Src family, and Ras in cardiac fibroblasts. Circ Res 1998;82:337-345. 